\title{
Design of Remote Gateway for Multi-Connected Inverter Air-Condition based on STM32
}

\author{
Xiao-dong Liu ${ }^{1,2}$,Ji-you Fei ${ }^{1}$ Hao Meng ${ }^{2}$, Xiao-dong Li $^{2}$,Long Wang ${ }^{2}$ \\ ${ }^{1}$ EMU Department, Dalian Jiaotong University; \\ ${ }^{2}$ Panasonic Appliances Air-Conditioning and Refrigeration (Dalian) Co. Ltd.; \\ Dalian, China \\ a) xiaotianli1993@163.com
}

Keywords: Remote gateway, Multi-Connected inverter, Air-Condition, STM32

\begin{abstract}
In order to meet the requirements for multi-connected inverter air-condition of diversified service, intelligent control and reliable operation, an embedded home gateway terminal system based on STM 32 signal chip microcomputer, WIFI network and RS485 communication technique has been designed and is presented in this paper. After acquiring the real-time data by using this gateway, the system can realize Big Data Analysis and faults diagnosis of air-condition. The system is an application of Internet of Thing, it can solve the problem of information sharing, and accommodate both the customers and manufactures' needs of monitoring and fault diagnosis for the whole cycle of air-condition.
\end{abstract}

\section{INTRODUCTION}

In recent years, the Multi-Connected Inverter Air-Condition (MACU) with the advantages of high effective energy conservation and environmental protection develops vigorously, and the technology of remote control air-condition has been gradually maturing. Big data analysis in AC field becomes more and more attractive. Mining data of abundant of AC data storage, the customers' behavior can be predicted, so by analyzing big data of AC, it can help to improve the AC system, diagnosis AC faults and prognostics the faults. The home terminal system for MACU is the key part to connect sensing network and the wide-area network. In order to shield the heterogeneity from internal arrangement of the sensing network, and to meet low-power and flexible manipulation requirements, provide a complete hardware foundation of big data analysis of AC, a design of embedded gateway air-condition with home terminal system based on Internet of Things is presented in this paper, according to the demand analysis, STM signal chip microcomputer is chosen to be the controller component.

\section{SYSTEM DESIGN}

The overall block diagram of the system structure is shown in figure 1 , the sensing notes of the Indoor Unit of Air Condition are distributed in a form of network.

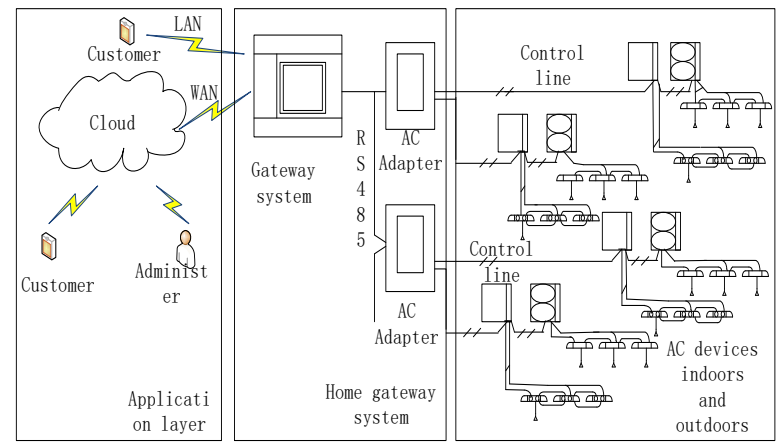

FIGURE 1 Overall block diagram of the system structure

With the special adapter, the data including running state, temperature and humidity of the indoor 
room can be collected into the control gateway, so the centralized inspection of operation state, energy consumption and circumstances can be realized. All function, like parameters setting, can be controlled individually. In addition, the thesis introduces the home terminal system of MACU, focus on remote controlling and shielding the heterogeneity, to fulfill the requirement of connecting the sensing network and mobile termination. Through the network to realize remote the monitoring function, human-machine interface well, the program also can be used to record information function for the system to conduct a comprehensive analysis, improve the system stability and reduce fault.

\section{THE STRUCTURE AND FUNCTION OF HARDWARE OF THE GATEWAY SYSTEM}

This paper studies the MACU, designs intelligent acquisition system with STM32F407 embedded technology. The system block diagram has been shown in figure 2. The hardware is composed by RS485 communication module, intelligent routing module and control terminal, which supplied by battery to meet the requirement of low-power consumption.

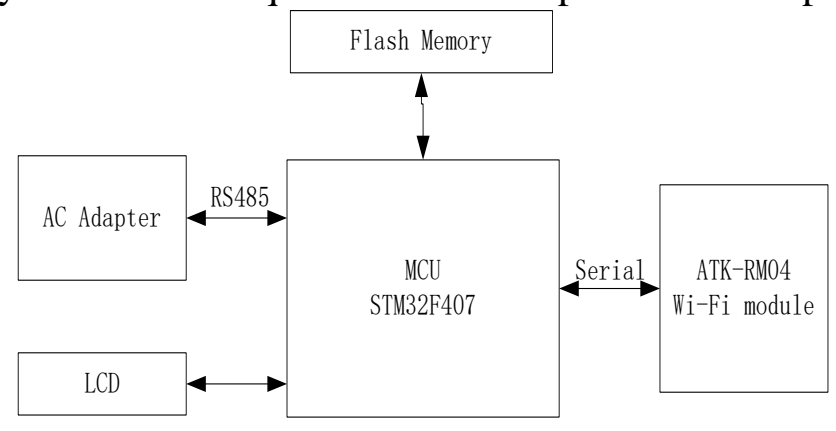

FIGURE 2 Control terminal block diagram

The hardware functions of the control termination are as follows:

Processor STM32F407. It is based on 32 bit ARM cortex-M3 kernel, 1M bytes Flash memory and 196K bytes SRAM. Its highest frequency can reach $168 \mathrm{MHz}$. It has the characteristics of high performance, low cost, low power consumption and high-speed operation.

RS485 communication module. By using RS485 communication module, the system can acquire information about AC running condition, circumstances parameters and transmit control instrument to the indoor room AC unit.

Intelligent routing module. There are two available communication models, so the AC terminal system can be monitored and controlled by Home LAN and WAN. The system is able to connect with cloud platforms, manage router and switch the mode of network operation. Due to the issues of interference and electromagnetic compatibility, the system is required for a better reliability and real-time response.

LCD. Display the temperature information measured by gateway system.

Flash memory. By recording the AC testing parameters into the control termination, the system can provides the data for improving the control system efficiency.

\section{SOFTWARE DESIGN AND ANALYSIS}

\section{The RS485 communication of AC advice}

STM32F407 signal chip microcomputer takes on the role of controller in MACU system. It is responsible for the data collection and state controlling in real time. In order to realize the communication between residential gateway and AC, a system, which using STM32F407 signal chip microcomputer as master station and the communication adapter as slave station, is discussed in this paper. The communication between master and slave station is based on S-NET protocol and RS485. The STM32F407 signal chip microcomputer is accessed to the bus network through RS485 interface, the A port of the RS485 is non-inverting input, while B port is inverting input. The modes of sending or receiving are determined by high or low voltage levels of input PG8. The system can parallel 32 AC adapters, its maximum transmission distance is 1200 meters with transmission speed of $100 \mathrm{Kbps} / 1200 \mathrm{~m}$. The flowchart is shown in figure 3 . 


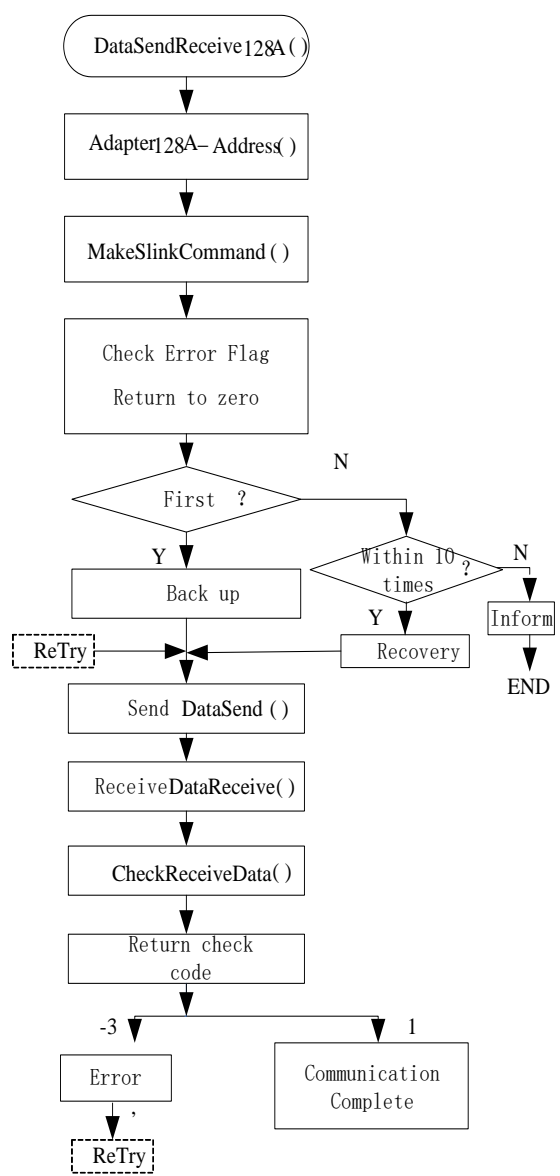

FIGURE 3 The flowchart of communication program

The structure of S-NET protocol concludes host querying and slave replying. In a communication processing, firstly, STM32F407 signal chip microcomputer will calculate the address of slave device (adapter), and then generate control commands, finally, send these commands to the slave device. There are several types of communication instruction, such as service type (including setting, operation, query, reply, condition modify and corresponding model etc.). So, different communication can be realized by using different types.

The physical layer is defined by RS485, the upper-layer protocol applies the S-NET protocol developed by some AC company to connect the masters and slaves in the system. The format of SNET protocol structure is shown in table 1 in details.

TABLE 1

THE FORMAT OF S-NET PROTOCOL STRUCTURE

\begin{tabular}{c|c|c|l|l|c}
\hline $\begin{array}{c}\text { source } \\
\text { address }\end{array}$ & $\begin{array}{c}\text { Destination } \\
\text { address }\end{array}$ & $\begin{array}{c}\text { Control } \\
\text { code }\end{array}$ & BC byte & $\begin{array}{c}\text { extended } \\
\text { address }\end{array}$ & $\begin{array}{c}\text { Sub- } \\
\text { source }\end{array}$ \\
\hline 1byte & 1byte & 1 byte & 1byte & 1 byte & 1 byte \\
\hline $\begin{array}{c}\text { Sub- } \\
\text { destination }\end{array}$ & $\begin{array}{c}\text { Sub- } \\
\text { extended } \\
\text { address }\end{array}$ & $\begin{array}{c}\text { random } \\
\text { number }\end{array}$ & $\begin{array}{c}\text { Command } \\
\text { code }\end{array}$ & Data & $\begin{array}{c}\text { FCC } \\
\text { Check }\end{array}$ \\
\hline 1byte & 1byte & 4 bit & 1byte & $1-256$ bit & 1byte \\
\hline
\end{tabular}

(1) Source address and destination address: to choose the specific device for sending or receiving commands.

(2) Control code: categorize the commands from host state, so the slave device can response with different functions.

(3) Byte number: to calculate the byte number of both commands and data.

(4) Commands and data: including the command or data information.

(5) FCC check code: identify the correctness of data based on parity check. 


\section{Intelligent routing module}

The Intelligent routing module access the wireless network by using RM-04 Wi-Fi module, communicate with MCU by series. It includes TCP/IP protocol stack, so it can realize series, Ethernet and Wi-Fi communication. The function chart is given in figure 4. When STM32 communicate with Wi-Fi module, the system applies DMA (Direct Memory Access) mode to acquire and receive data. It is a fast data transfer operating mode through which outside device and memory can write and read data each other directly without the temporary store of CPU.

The TCP/IP server/client model is build based upon the TCP/IP protocol stack which can be used as network layer and transport layer. Every home can be equipped with a intelligent remote AC control termination, which used to be the TCP service, so it can send condition information to and receive commands from the PC and various mobile APP clients. The communication structure is mainly composed of physical layer, data link layer, network layer and transport layer. The physical layer and data link layer are using IEEE802.11b standards, the carrier frequency is $2.4 \mathrm{GHz}$, the traffic rate is $11 \mathrm{Mbit} / \mathrm{s}$. Aiming to meet the need of data -rate transmission, a approach bandwidth is set. Network and TCP service are using TCP/IP protocol, the IP address of intelligent termination is random allocation, and the subnet mask is 255.255.255.0. Using the acquisition board as TCP service, its port is 4000 .

TABLE 2 TABLE OF FRAME FORMAT

\begin{tabular}{c|c|c|l|l}
\hline Flag & Frame number & Customer data and commands & CRC check & Flag \\
\hline 0x7E & 1byte & N byte & 2byte & 0x7E \\
\hline
\end{tabular}

0X7E (1byte) has been used as the flag, once 0X7E (1byte) occurs in frame number, customer data or CRC check, it must be processed with following rules:

$0 \times 7 \mathrm{E}<\longrightarrow 0 \times 7 \mathrm{D}$ follow closely with $0 \times 02$;

$0 \times 7 \mathrm{D}<\longrightarrow 0 \times 7 \mathrm{D}$ follow closely with $0 \mathrm{x} 01$;

The processing procedures are introduced as following:

During sending a command: frame forming $\rightarrow$ data verification $\rightarrow$ escaping

During receiving a message: Reversal escaping $\rightarrow$ data verification $\rightarrow$ Extract information

For example: sending a message, the original data will change from 0x20 0x7D 0xF8 0x7E 0x55 into 0x7E 0x20 0x7D 0x01 0xF8 0x7D 0x02 0x55 0x7E after packaging.

The frame number range from 0 to 124,124 equal to $0 x 7 C$ in hex. Once the TCP communication has been build or the frame number is over 124, the frame number will return to zero. To make sure the system not missing any frame, a method of ARQ has been applied during data transmission. Because sampling interval is within 2s, the speed of acquisition data is relatively slow; only one frame will pass the data window each time, this frame of data won't be accepted if there is no reply. Check code is calculated based on CRC-16 check rules, located closely behind customer data.

In order to ensure the reliability and validity of the communication between the wireless remote controller and the AC termination, the system will check the frame transmission overtime or not. Once the receiver indented the signal of $0 x 7 \mathrm{E}$, it will change into receiving mode, setting $10 \mathrm{~ms}$ time, if after $10 \mathrm{~ms}$, the receiver didn't get a signal of another 0x7E, system will give notice that there is overtime. Then the receiver will abandon the data, and wait for new loop to receive data. Once the receiver has got all the data, it will calculate the amount of the frame, if its length is less than five, the receiver will abandon the data. Take the remote AC into account, if the customer wants to set temperature, the control termination will receive a command composed as form as Table 3.

TABLE 3 FRAME STRUCTURE OF TEMPERATURE CONTROL COMMAND

\begin{tabular}{c|c|c|c|c}
\hline Flag & Frame number & Commands & $\begin{array}{c}\text { Indoor } \\
\text { device } \\
\text { number }\end{array}$ & $\begin{array}{c}\text { Indoor } \\
\text { Controlled } \\
\text { Machine }\end{array}$ \\
\hline 0X7E & 1byte & C2 & $\begin{array}{c}\text { 1byt } \\
\text { e }\end{array}$ & 8byte \\
\hline Model setting & Temperature setting & CRC check & Flag & \\
\hline 1byte & 1byte & 2byte & 0X7E & \\
\hline
\end{tabular}


The frame number represents the sequence number, 0XFF represents all frame of one command has been transmitted successfully, the system need to be prepared for the next coming data. The processing of communication program is: connect firstly, then enable interrupts, once the gateway system receive command, the system will separate data, and send the different data to corresponding AC equipment, finally, the real-time information of condition will be returned and updated. It is tested that the transmit platform of the wireless network meets the actual need of the system.

\section{CONCLUSION}

This paper introduced a embedded AC control gateway system based on STM32 signal chip microcomputer, Wi-Fi Network and RS485 communication technology. An Internet connection mode based on the S-NET protocol is analyzed. The system can shield the heterogeneity from internal arrangement of sensing network and operate in low power. Trough monitoring and controlling the AC device based Internet of Things, this thesis intends to use for further studies of the smart home and intelligent device. In addition, this design can provide a complete hardware foundation for AC data mining and analysis.

\section{ACKNOWLEDGMENT}

This work was financially supported the funds from Liaoning Education Department (serial number: JDL2016026) and the Doctoral Research Fund from Liaoning Province (serial number:201601259).

I would like to express my heartfelt gratitude to my supervisor, for his constant encouragement and guidance. He put forward many valuable revision opinion. And I would like thank to my classmates and senior, thank for them selfless help my writing.

\section{REFERENCES}

[1] Yuan, Hua, et al. "Design and implementation of Android-based intelligent remote controller for the video conferencing terminal."Journal of Guangxi University(2011).

[2] Liu Qunqun. Research and Development of Intelligent Air Conditioning Based on Mobile Internet [D]. South China University of Technology . 2013.

[3] LI Xiaodan. Design of IOT embedded gateway based on STM32 [J]. Computer Engineering and Applications. 2015, (4).

[4] Dual mode cloud air conditioning system based on Wi-Fi communication mode [P]. CN 103916464 A), 2014.07.09.

[5] Lee T S, Tseng K H, Chen L D, et al. Intelligent air-condition system: US, US6715689[P]. 2004.

[6] Bernaden, Iii, and Alex. "State machine controller for operating variable air volume terminal units of an environmental control system." (2001).

[7] Xu, Hongyan, G. Wu, and C. Chen. "Based on STM32F103 implement Profibus-DP slave with high-speed transmission."International Conference on Uncertainty Reasoning and Knowledge EngineeringIEEE,2011:232-234.

[8] Liu, F., and H. Zhao. "The Design of WIFI-Based Smart Home Communication Hardware Adapter." International Conference on Instrumentation \& Measurement 2015:1193-1197. Chan, Ping Chieh, et al. "Intelligent control method for air condition device." (2016).Article in a conference proceedings

[9] Yuan, Jie, and T. H. Chang. "Design of ultrasonic distance measurement system based on STM32 microprocessor."Electronic Design Engineering(2011).

[10] Chen Huanxin, Liu Jiangyan, Hu Yunpeng, Li Guannan. Application of Big Data in Air- 
conditioning Field[J]. Journal of Refrigeration. August,2015.Vol.36,No.4:16-22.

[11] Manyika J, Chui M, Brown B. Big data: the next fromtier for innovation, competition, and productivity[M]. Mckin-sey Global Institute, 2011.

[12] LI Li, LIU Chong, MIAO Zhong-Hua, ZENG li. A Networked Test System of Multi Inverters and IPC Based on RS485[J]. Measurement and control technique. 2016: 35(7)

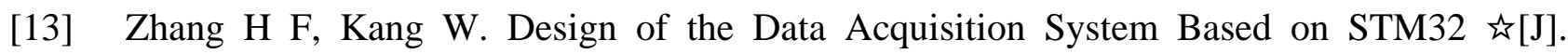
Procedia Computer Science, 2013, 17(Complete):222-228.

[14] Lei, Xu, Guan, et al. Design on Remote Intelligent Terminal Unit of Industrial Ethernet Based on STM32[J]. Advanced Materials Research, 2012, 466-467:1167-1171.

[15] Zhang X. Design of electric power data acquisition system based on STM32[J]. Electronic Measurement Technology, 2010.

[16] Hu J L, Zeng S, Zhang Z. The Design of Wireless Data Acquisition System Based on STM32 and Virtual Instrument[J]. 2012:1-4.

[17] Corporation G I. Enhancing targeted advertising in a home network gateway device[J]. 2014.

[18] Telegraph N, Corporation T. DOMESTIC NETWORK SETTING METHOD, HOME GATEWAY DEVICE, HOME GATEWAY PROGRAM, AND RECORDING MEDIUM[J]. 2007.

[19] Saito T, Tomoda I, Takabatake Y, et al. Home gateway architecture and its implementation[J]. IEEE Transactions on Consumer Electronics, 2000, 46(4):1161-1166.

[20] Kushiro N, Suzuki S, Nakata M, et al. Integrated home gateway controller for home energy management system[C]// Consumer Electronics, 2003. ICCE. 2003 IEEE International Conference on. IEEE, 2003:386 - 387. 